\title{
ACUTE MYELOID LEUKEMIA-M3V WITH DEEP VEIN THROMBOSIS, A RARE PRESENTATION
}

P. Mani Mekhalaํ, M. Lavanya², L. Aruna ${ }^{3}$, Shajia Rehman4, Pooja Deshmukh ${ }^{5}$

\section{HOW TO CITE THIS ARTICLE:}

P. Mani Mekhala, M. Lavanya, L. Aruna, Shajia Rehman, Pooja Deshmukh."Acute Myeloid Leukemia-M3v with Deep Vein Thrombosis, A Rare Presentation". Journal of Evolution of Medical and Dental Sciences 2014; Vol. 3, Issue 02, January 13; Page: 241-245, DOI:10.14260/jemds/2014/1816

ABSTRACT: Early diagnosis of acute promyelocytic leukemia (APL) is essential because of its associated life threatening coagulopathy. Among patients with acute leukemia, incidence of thrombosis is highest in acute promyelocytic leukemia (APL) In APL many variants have been identified, hypergranular and hypogranular variant. Herewith we report a case of 27 year old male presenting with non-specific symptoms like fever, SOB, cough with hemoptysis, swelling right lower limb and was found to have deep vein thrombosis. It is important to identify the hypogranular variant as it poses a diagnostic challenge.

KEY WORDS: APL, AMLM3v, DVT, Hypogranular AML.

INTRODUCTION: Acute promyelocytic leukemia(APL, FAB-M3) accounts for about 5 to $10 \%$ of patients with AML, characterised by neoplastic proliferation of promyelocytes and blasts ${ }^{1}$. Median age of presentation is 30 to 38.The disease was recognised in the 1950 s with up to $90 \%$ of cases presenting with hemorrhagic manifestations secondary to DIC ${ }^{2}$. Thrombotic complications in acute leukemia are often underestimated because bleeding complications generally dominate the clinical picture ${ }^{1}$.

Here we present a case of APL, hypogranular variant presenting with deep vein thrombosis in the right lower limb. It is a rare presentation and is difficult to treat due to risk of haemorrhage and thrombotic complications.

CASE SUMMARY:A 27 year old male presented to our hospital with complaints of high grade fever, shortness of breath, cough with expectoration, and swelling of the lower limb since 6 days. There was also associated haemoptysis. On examination the patient was dyspnoeic with cellulitis of the right lower limb. There was no lymphadenopathy or hepatosplenomegaly.

\section{LABORATORY FINDINGS:}

Blood picture: His initial blood counts showed haemoglobin $5.3 \mathrm{~g} / \mathrm{dl}$ with microcytic hypochromic pictureTLC-23000/ $\mu$ l, platelets $1,11,000 / \mu$ l with predominantly blasts. His bilirubin was 1.1 , SGOP16U/L, SGPT-300U/L, ALP-215/L, blood urea-40U/L, Serum creatinine-0.9mg/dl. His chest x-ray was normal. In view of deep vein thrombosis (DVT), he was investigated for D-dimers which were markedly elevated to1240ng/ml and PT-16 and INR-1.22.As the peripheral smear showed blasts, with a high index of suspicion of a haematological malignancy his repeat haemogram was done which showed $\mathrm{Hb}-8.5 \mathrm{~g} / \mathrm{dl}$, TLC- $16500 / \mathrm{ml}$ with $88 \%$ blasts with 1.5 to 2 times the size of lymphocyte with scant to moderate cytoplasm with round to indented to bilobed nucleus with coarse chromatin with 1-2nucleoloi with very fine granules in the cytoplasm. Doppler study was suggestive deep vein thrombosis in the common femoral vein, saphenofemoral vein, deep femoral vein, popliteal vein. 
Ultrasonography of abdomen was normal. A tentative diagnosis of acute myeloid leukaemia, possibly APL was given. The marginal raise of total leukocyte count and not very distinct granules and varied presentation posed a diagnostic dilemma, however we proceeded for further investigations like bone marrow aspiration and cytochemistry.

Bone marrow: Bone marrow aspiration was done from sternum at the bedside as the patient was dyspneic. The findings were:-markedly hypercellular bone marrow, with markedly suppressed erythropoiesis and megakaryopoeisis. Granulopoeisis was markedly increased with promyelocytes, myeloblasts being predominant nucleated cells in the bone marrow. Blasts showed scant to moderate cytoplasm with 1-2 nucleoli with scant dust like granules. Few blasts showed cytoplasmic blebs and promyelocytes also showed clefted nucleus and bilobed nucleus-suggestive of Acutemyeloid leukaemia -FAB M3v (hypogranular/ microgranular).

Cytochemistry: Myeloperoxidase was positive in $60 \%$ of blasts.

Immunohistochemistry: As the patient expired in the immediate period we submitted the bone marrow aspiration cell block for Immunohistochemistry. It was found that CD117 was positive and CD34 was negative in most of the blast cells.

DISCUSSION: The association of thrombosis and malignancy has been long studied since it was first described by Trousseau; however there is a paucity of data that pertain to patients with hematologic malignancies. The results of a large population-based study indicate that lower extremity deep vein thrombosis, upper extremity deep vein thrombosis, or pulmonary embolism occurred frequently in patients with either AML or ALL, particularly in the first 3 months ${ }^{3}$. Among the malignancies, incidence of thromboembolism is high in lymphomas or acute leukemia ${ }^{4}$.Thrombosis can be a presenting symptom at diagnosis in 9.6\% of APL cases and 3.2\% in non-M3 AML; a similar rate of thrombosis can occur during the subsequent course of the disease ${ }^{5}$.Thromboembolism is often overshadowed in acute leukaemia due to increased incidence of bleeding and infections. Acute Myeloid Leukemia is a morphologically and genetically heterogenous disease. One subtype with specific morphology and specific cytogenetic and molecular genetic aberration is acute promyelocytic leukemia (APL). On the basis of morphology alone, APL can be separated into two distinct subtypes according to FAB classification: AML M3 and AML M3 variant (AML M3 v).The latter is also called microgranular (hypogranular) APL in new WHO classification. The cytomorphology of APL blasts is obviously different in the two subtypes: in AML M3, the abnormal promyelocytes show heavy granulation and bundles of Auer rods; in AML M3v, blasts have agranular or hypogranular cytoplasm or contain fine dust like cytoplasmic granules that may sometimes be unclear on light microscopy. Furthermore, M3v blasts show a typically bilobed nuclear configuration. This latter morphology, together with missing granulation, often resulted in a misleading diagnosis of acute monocytic or myelomonocytic leukemia 6 .Flowcytometry of Hypogranular (microgranular) APL is characterized by low Side Scatter or SSC and moderate expression of CD45 (leukemic cells distributed in the "blast" region on CD45 versus SSC dot plot display, similar to blasts in non-APL acute myeloid leukaemia) ${ }^{7}$. Neoplastic cells in this variant were positive for CD2 (80\%), CD4 (30\%), CD13 (95\%), CD33 (100\%), CD34 (75\%), and CD117 (100\%), whereas HLA-DR, CD10, CD11b, and CD11c were always negative.

This case report emphasizes the presentation of venous thromboembolism (VTE) in acute leukaemia especially in a hypogranular variant of APL where morphological diagnosis over 


\section{CASE REPORT}

peripheral smear and bone marrow is difficult due to the fine dust like azurophilic granules admixed with clefted and bilobed cells with an occasional entrapped neutrophil and hence stresses upon high index of suspicion in such a presentation. One of the nearest differentials for AML-FABM3v is AMLFABM5 especially as it presents as VTE. According to Amayliaetal there was 6.3\% overall incidence of thrombosis, mostly involving the venous vessels ( $80 \%$ of the cases); $3.4 \%$ of all the patients suffered from thrombosis as one of the heralding manifestations of the disease, with a higher incidence among patients with APL $(9.6 \%)^{4}$. According to Imberti $11.4 \%$ of VTE incidence in acute leukemia cases and of which 50\% were AML M2 and M5 and 50\% were ALL9.

To conclude we present this case to reiterate that proper and early recognition with a high index of suspicion of APL-FABM3v in a case presenting with VTE aids in early treatment and to warrant the incidence of VTE while treating with ATRA. However in our case the patient succumbed to DVT and its complication soon after diagnosis.

CONCLUSION: Thromboembolic complications often dominate the clinical presentationin acute leukemia, here we emphasize the presentation of acute leukemia as DVT since the identification of acute leukemia especially APL-FAB M3v is important as morphological diagnosis is difficult and needs high index of suspicion and differentiation from AML-FAB M5.

\section{REFERENCES:}

1. Kafil Akhtar, Shamshad Ahmad, Rana K. Sherwani.Acute promyelocytic leukemia, hypogranular variant: a rare presentation.Clinics and Practice.2011;vol 1.p. 1:e11.

2. Ted Wun.Venous thromboembolism in patients with acute leukemia, lymphoma, and multiple myeloma. Elsevier Ltd., April 2010. Papers and Abstracts of the 5th International Conference on Thrombosis and Hemostasis Issues in Cancer. pp. Volume 125, Supplement 2, Pages S96S102.

3. Grace H. Ku, Richard H. White, Helen K. Chew etal. Venous thromboembolism in patients with acute leukemia: incidence, risk factors, and effect on survival.Blood. 2009 April 23; 113(17): 3911-3917.

4. Amaylia Oehadian, Mohammad Iqbal, Rachmat Sumantri. Deep vein thrombosis in acute myelogenousleukemia. ActamedicaIndonesiana.Source: PubMed10/2009; 41(4):200-4.

5. De Stefano V, Sora F, Rossi E, Chiusolo P etal. The risk of thrombosis in patients with acute leukemia: occurrence of thrombosis at diagnosis and during treatment. J Thromb Haemost. 2005; 3. 1985-1992.

6. Torsten Haferlach, Alexander Kohlmann, Susanne Schnittgeretal. AML M3 and AML M3 Variant each have a distinct gene expression signature but also share patterns different from other genetically defined AML subtypes. Genes, Chromosomes\& Cancer. 2005; 43:113-127.

7. Wojciech Gorczyca etal. Acute promyelocytic leukemia: four distinct patterns by flow cytometry immunophenotyping. Pol J Pathol.2012; 1: 8-17.

8. Nargund AR, Patil GV, Raghuram C P, Venkataswamy E. CD34 positive-microgranular variant of acute promyelocyticleukemia in a child. Indian J PatholMicrobiol. 2012; 55:574-7

9. Imberti D, Vallisa D, Anselmi E et al. Safety and efficacy of enoxaparin treatment in venous thromboembolic disease during acute leukemia.Tumori. 2004; 90:390-3. 


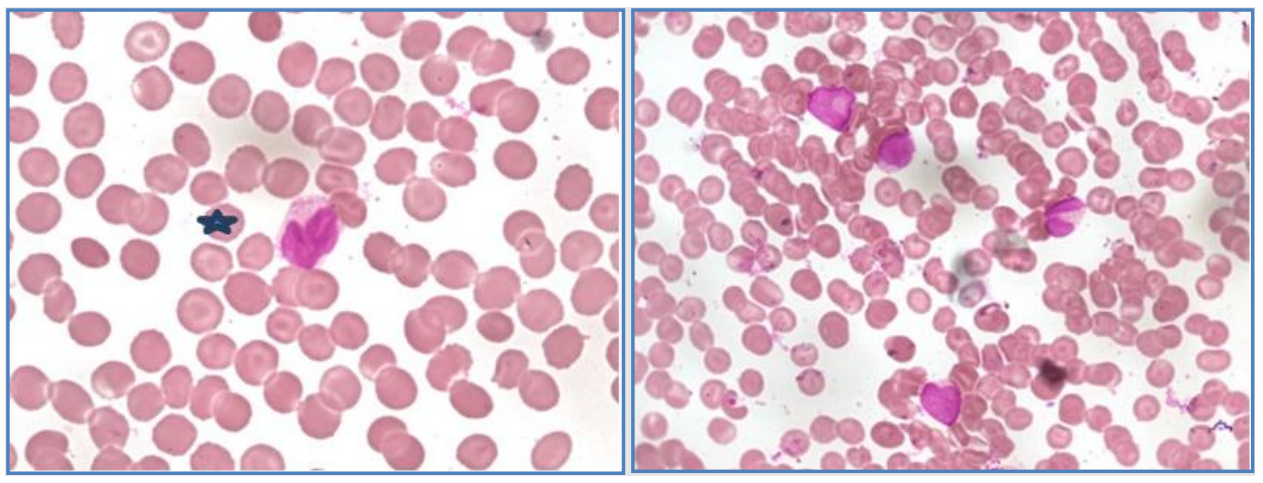

Fig. 1: photo micrograph $(40 \mathrm{X})$ of peripheral smear showing the bilobed or convoluted nucleus of blast few showing dust like fine granules in AML M3v.

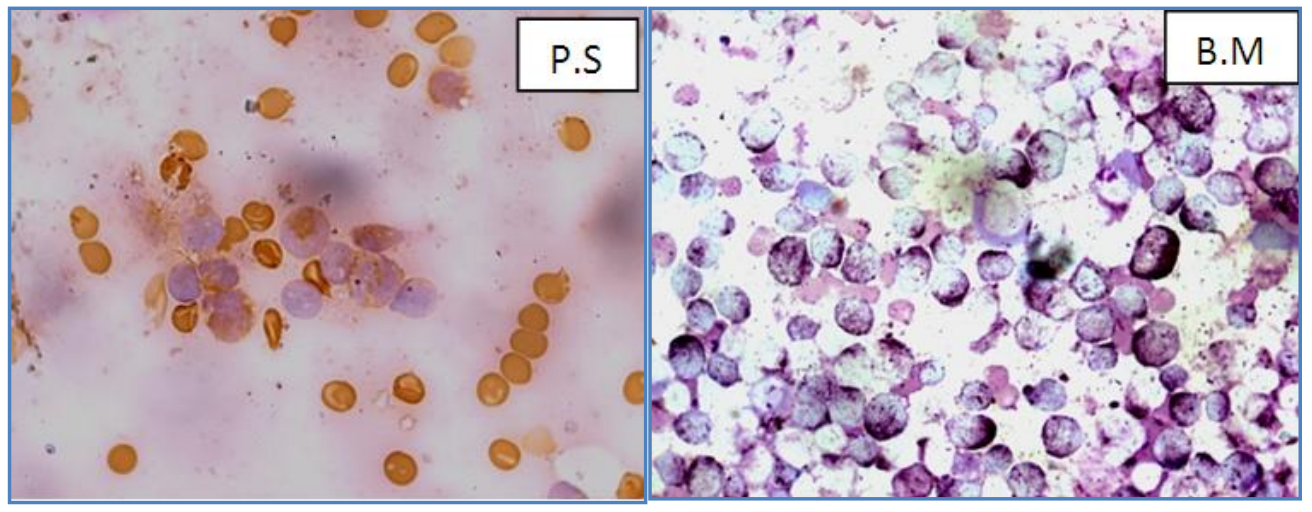

Fig. 2: Photomicrograph showing Myeloperoxidase staining showing positivity in blasts.
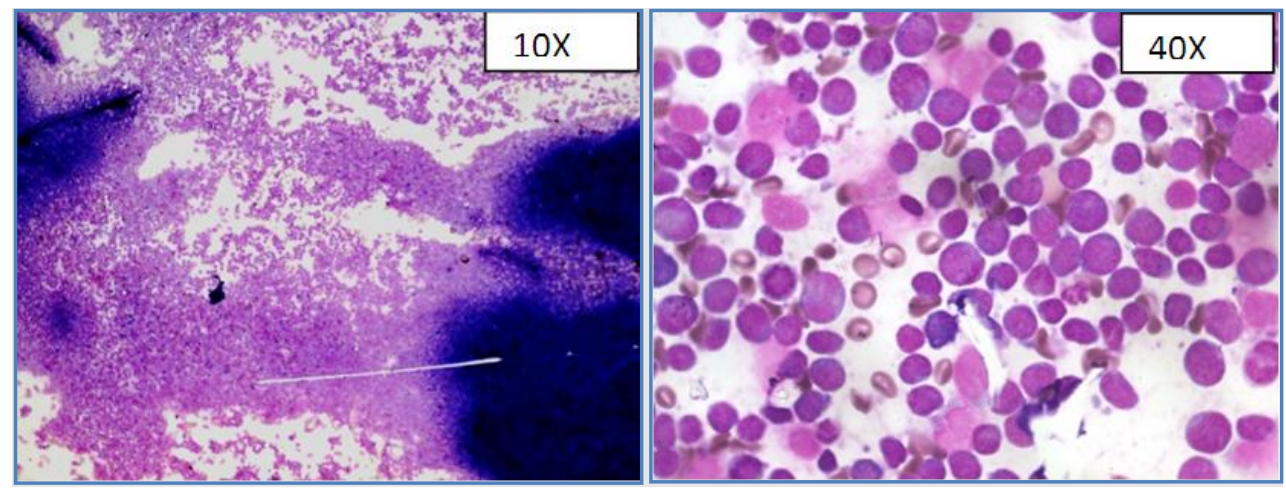

Fig. 3: photomicrograph showing hypercellular bone marrow and many blasts 


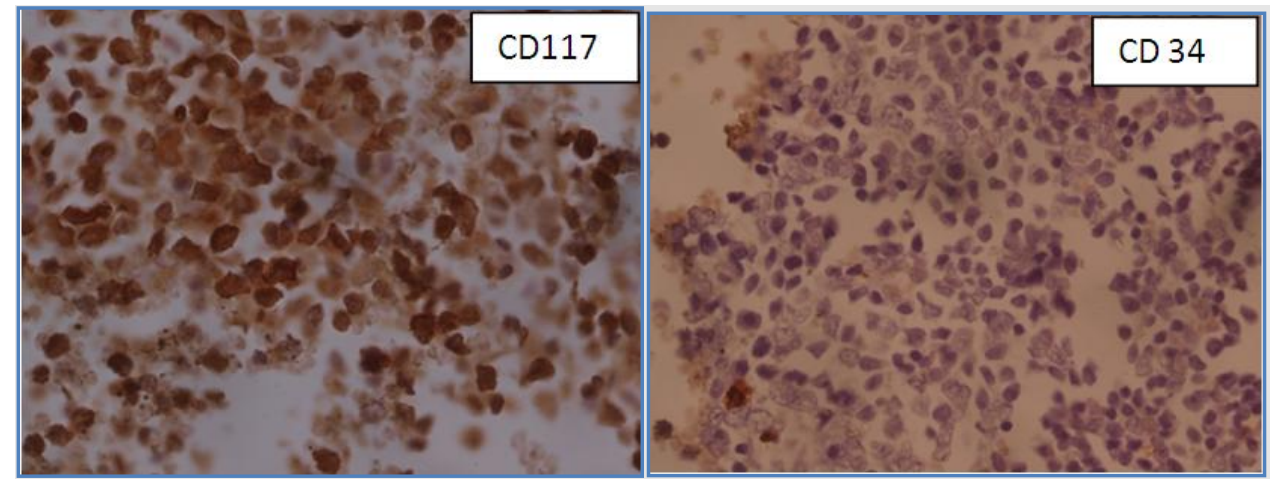

Fig. 4: Photomicrograph of bone marrow aspiration cell block Showing CD117 positivity and CD34 negativity

\section{AUTHORS: \\ 1. P. Mani Mekhala \\ 2. M. Lavanya \\ 3. L. Aruna \\ 4. Shajia Rehman \\ 5. Pooja Deshmukh}

\section{PARTICULARS OF CONTRIBUTORS:}

1. Associate Professor, Department of Pathology, Osmania General Hospital, Hyderabad.

2. Assistant Professor, Department of Pathology, Osmania General Hospital, Hyderabad.

3. Assistant Professor, Department of Pathology, Osmania General Hospital, Hyderabad.

4. Post Graduate, Department of Pathology, Osmania General Hospital, Hyderabad.
5. Post Graduate, Department of Pathology, Osmania General Hospital, Hyderabad.

\section{NAME ADDRESS EMAIL ID OF THE CORRESPONDING AUTHOR:}

Dr.P. Mani Mekhala,

Associate Professor,

Department of Pathology,

Flat No. 204, A Block, Vishnu Residency,

Gandhi Nagar, Hyderabad.

Email-drmanimekhala@gmail.com

Date of Submission: 18/12/2013.

Date of Peer Review: 19/12/2013.

Date of Acceptance: 31/12/2013.

Date of Publishing: 07/01/2014 